\title{
Navegación remota en la fibrilación auricular
}

\section{Remote navigation in atrial fibrillation}

\author{
Jorge Eduardo Marín-Velásquez ${ }^{a, b, c}$, Julián Miguel Aristizábal-Aristizábal a,b,c,d, \\ Jorge Enrique Velásquez-Vélez ${ }^{\mathrm{a}, \mathrm{b}, \mathrm{c}, \mathrm{e}, \mathrm{f}}$, Mauricio Duque-Ramírez ${ }^{\mathrm{a}, \mathrm{b}, \mathrm{c}, *}$, \\ Juan Carlos Díaz-Martínez ${ }^{a, b, c}$ y William Uribe-Arango ${ }^{a, b, c, f}$
}

\author{
a Universidad CES, Medellín, Colombia \\ b Clínica CES, CES Cardiología, Medellín, Colombia \\ c Electrofisiología Clínica Las Américas, Medellín, Colombia \\ d Clínica del Rosario, Medellín, Colombia \\ e Clínica Somer, Rionegro, Colombia \\ ${ }^{f}$ Centros Especializados de San Vicente Fundación, Medellín y Rionegro, Colombia
}

Recibido el 3 de octubre de 2016; aceptado el 6 de octubre de 2016

Disponible en Internet el 5 de noviembre de 2016

Se presentan los sistemas de navegación remota de uso más "común" en el mundo para uso en ablación fibrilación auricular. Por ser una tecnología que no tiene suficiente expansión y uso clínico, todavía no existen guías que recomienden su uso; solo hay reportes de trabajos no aleatorizados en los centros donde están disponibles.

La ablación con catéter para aislar las venas pulmonares se ha convertido en el tratamiento de elección para la fibrilación auricular paroxística sintomática refractaria al tratamiento farmacológico. El objetivo de la ablación con catéter por radiofrecuencia (RF) es lesionar los sustratos arritmogénicos mediante la entrega de grandes cantidades de energía al tejido cardíaco, impidiendo la conducción eléctrica y eliminando así la arritmia. La ablación con catéter de radiofrecuencia se considera como un tratamiento eficaz y se recomienda como tratamiento de primera o segunda línea de acuerdo con las últimas guías ${ }^{1}$.

Recientemente, varias estrategias se han desarrollado para aumentar la eficacia de ablación de la fibrilación auricular ${ }^{2}$.

\footnotetext{
* Autor para correspondencia.

Correo electrónico: mauricioduque@une.net.co (M. Duque-Ramírez).
}

Estas técnicas se basan principalmente en una variedad de métodos para obtener el aislamiento de las venas pulmonares. Esta estrategia incluye la creación de líneas de bloqueo para inhibir la conducción eléctrica entre la aurícula izquierda y las venas pulmonares. Sin embargo, para el tratamiento de la fibrilación auricular persistente donde el aislamiento de venas pulmonares por sí solo no es suficiente, también se llevan a cabo ablación extensa lineal de la aurícula izquierda o ablación de electrogramas auriculares fraccionados complejos. Ambos enfoques requieren precisión y estabilidad del catéter de ablación.

\section{Justificación de la robótica: la eficacia y la seguridad}

La cartografía o mapeo precisos y la ablación de la zona de interés pueden ser complejos. Incluso para los operadores experimentados, los procedimientos pueden ser muy desafiantes y deben superarse múltiples dificultades para lograr un resultado exitoso y sin eventos adversos innecesarios ${ }^{3}$. Los requisitos cruciales para el éxito de la ablación en fibrilación auricular son: maniobrabilidad del catéter a las regiones de destino, estabilidad del catéter en situaciones 
anatómicas difíciles y reproducibilidad de la ubicación del catéter ${ }^{4}$.

Los catéteres manuales están limitados en su libertad de movimiento por su curva predefinida. En ciertas situaciones anatómicas, esto hará que algunas maniobras dentro del corazón sean extremadamente difíciles, y en ocasiones, las regiones anatómicas de interés no pueden ser alcanzadas en absoluto.

Teniendo en cuenta la importancia de la fuerza de contacto durante la ablación con catéter, los enfoques manuales tienen algunas limitaciones. Los operadores no siempre son capaces de hacer una estimación adecuada de la fuerza de contacto que se ejerce durante la ablación, lo que provoca diferentes fuerzas que se aplicarán entre las zonas de destino en las venas pulmonares y aurícula izquierda. (excepto con el uso de catéteres que tienen incorporado sensor de presión). Algunos resultados sugieren que las fuerzas más altas se aplican en la posición inferior septal derecha, y donde se aplica la fuerza más baja durante la ablación en la región inferior anterior izquierda y en la posición superior septal derecha ${ }^{5}$. Para el istmo mitral, proporcionar suficiente fuerza de contacto puede ser difícil debido a los movimientos continuos de la válvula. Puesto que la adecuada fuerza de contacto está relacionada con lesiones de ablación eficaces, las fuerzas más bajas darán como resultado un menor número de lesiones trasmurales ${ }^{6}$. Estas líneas de ablación inadecuadas serán sitios predominantes de reconducción o de aparición de nuevas arritmias macroreentrantes ${ }^{7}$.

Además de los problemas de maniobrabilidad, la manipulación de un catéter manual de mayor rigidez puede tener un alto riesgo de complicaciones. Cuando se mueve el catéter, a menudo se ejercen altas fuerzas de contacto y pueden estar en riesgo de perforar la pared auricular. Pese a que el taponamiento es una complicación con una incidencia global del $1-1,5 \%$, es la complicación mayor más frecuente ${ }^{8,9}$. No obstante, la ablación de fibrilación auricular es el procedimiento electrofisiológico más realizado hoy en día y la tasa de complicaciones sin duda tiene que disminuir.

Hay en la actualidad varios sistemas de navegación o mapeo y ablación robótica disponibles en el mercado, dentro de los cuales cabe destacar:

\section{Guiadas mecánicamente (Robots)}

1. Amigo $^{\circledR}$ (Catheter Robotics)

2. Sensei ${ }^{\circledR}$ (Hansen-Medical)

Amigo $^{\circledR}$ (Catheter Robotics) (figs. 1 y 2): este es un dispositivo robótico de control de catéteres a distancia que consta de dos partes: sistema de catéter remoto y controlador remoto. Este sistema tiene una vaina robótica para dirigir los catéteres que se controlan en una estación de trabajo cercana, de manera similar al sistema Sensei ${ }^{\circledR}$. El primer uso de este sistema en humanos data de 2010, en Leicester, Reino Unido, donde se empleó para realizar una ablación de aleteo auricular. De un uso poco extendido, su aplicación en aislamiento de venas pulmonares es muy limitado aun y la experiencia clínica la constituyen reporte de $\operatorname{casos}^{10}$. Se esperan más estudios para confirmar su seguridad y eficacia.

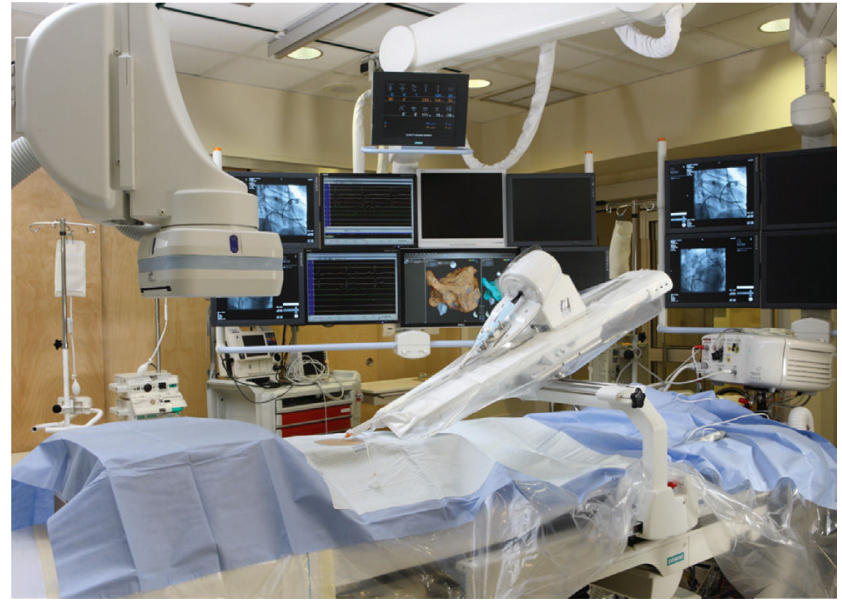

Figura 1 Disposición del sistema de navegación robótica en la sala de Electrofisiología.

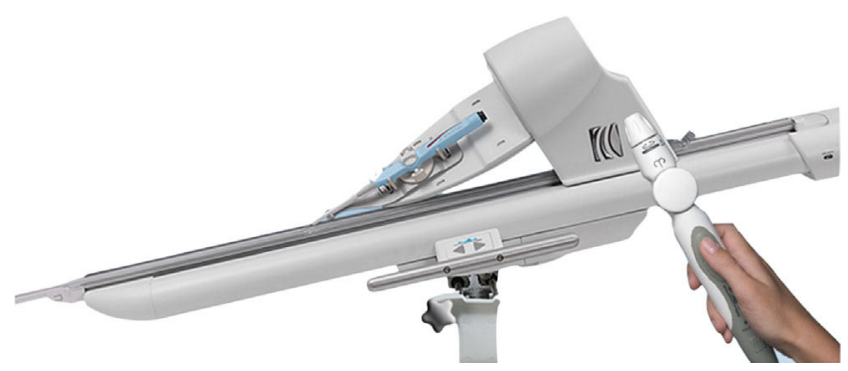

Figura 2 Sistema completo Amigo ${ }^{\circledR}$ (Catheter Robotics).

Sensei ${ }^{\circledR}$ (Hansen-Medical)(figs. 3 y 4): en 2010 recibió aprobación para iniciar ensayos clínicos y la exención como dispositivo de investigación.

El catéter Artisan $^{\circledR}$ tiene dos segmentos controlados robóticamente que proporcionan hasta 270 grados de articulación curva, que ayuda en el acceso de sitios de anatomía difícil. Varios centros han reportado tasas de éxito aguda y a largo plazo, que concuerdan con los procedimientos manuales $^{11-13}$. Dado que no existen ensayos controlados aleatorios terminados, que comparen la ablación robótica contra la ablación manual, emitir conclusiones sigue siendo difícil. Las técnicas, las tasas de complicaciones y los resultados clínicos de los procedimientos manuales y robóticos,

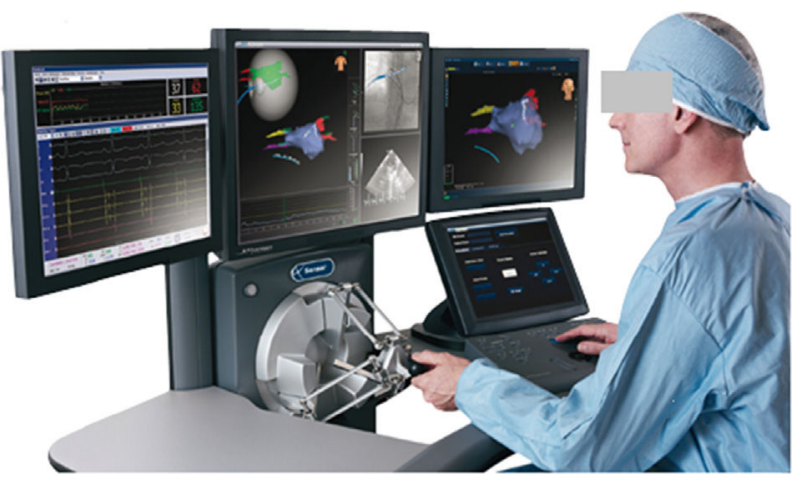

Figura 3 Control del brazo robótico del sistema Sensei ${ }^{\circledR}$ y extremo distal del catéter Artisan $^{\circledR}$ Reproducción de Hansen ${ }^{\circledR}$ Medical. 


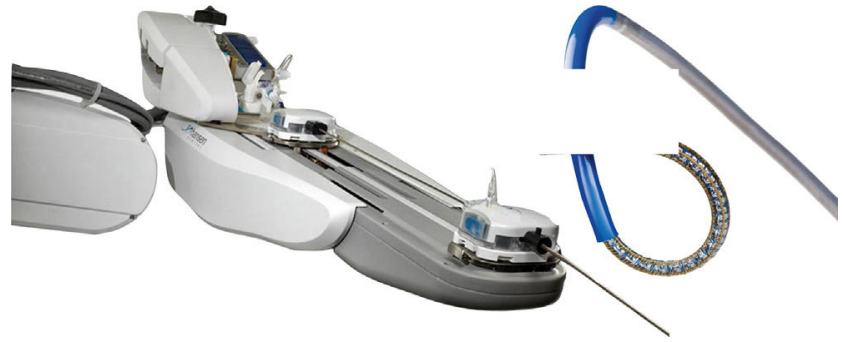

Figura 4 Sistema de mando a distancia con control tridimensional que permite el avance, retroceso, movimientos laterales y torsión del catéter de ablación. Reproducción de Hansen ${ }^{\circledR}$ Medical.

varían ampliamente entre los centros y esto hace que los datos del registro sean una tarea ardua de comparar.

\section{Guiadas magnéticamente}

1. Niobe $^{\circledR}$ (Stereotaxis)

2. $\mathrm{CGCl}^{\circledR}$ (Magnetecs)

\section{Navegación magnética para la ablación de fibrilación auricular: seguridad, eficacia y eficiencia}

El sistema de navegación magnética ((SNM); Niobe II, Estereotaxis Inc., MO, EE.UU.) es una tecnología de control del catéter remota, que puede proporcionar ventajas para prevenir complicaciones y mejorar la formación de la lesión. El SNM se compone de dos imanes externos situados en ambos lados del paciente, que generan un campo magnético $(0,08$ a $0,1 \mathrm{~T})$ dentro del paciente (fig. 5).

Un catéter magnéticamente activado atraumático (Biosense Webster Inc., CA, EE.UU. o Biotronik, Berlín, Alemania) incorpora cuatro imanes en el segmento distal que permiten que el catéter sea manipulado a distancia por los campos magnéticos direccionales. El operador puede alterar el vector del campo magnético y la punta del catéter se alineará con el vector, de manera que el operador podrá navegar la punta distal del catéter. El SNM permite el almacenamiento de vectores magnéticos para el acceso repetido mientras el catéter magnético navega automáticamente. El avance y la retracción del catéter se controlan

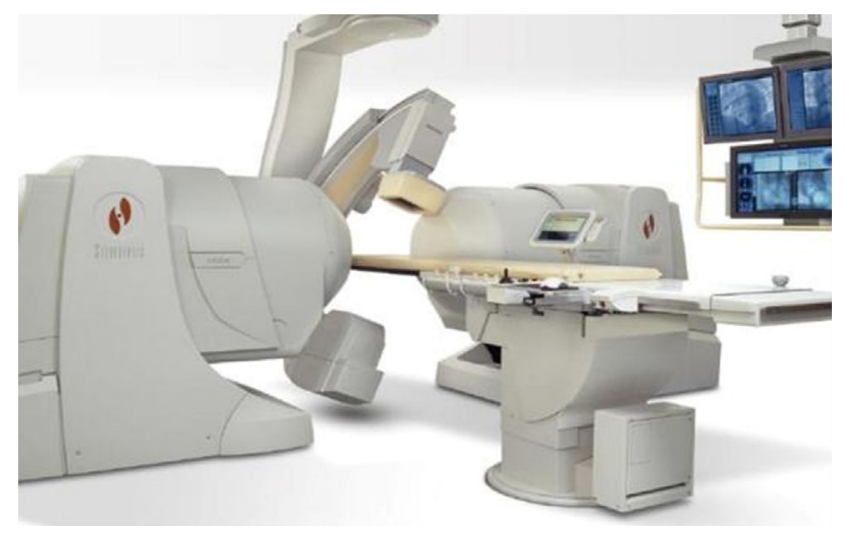

Figura 5 Sistema Niobe ${ }^{\circledR}$ II. Observe los grandes imanes al lado de la camilla. Reproducido con permiso de Stereotaxis ${ }^{\circledR}$ Inc. por separado mediante un accionamiento motor palanca de mando controlado (Cardiodrive, Stereotaxis Inc.). Las imágenes de los sistemas de cartografía 3D, fluoroscopia en tiempo real y tomografías computarizadas se pueden integrar completamente, lo cual facilita los procedimientos de cartografía y ablación totalmente controlados de manera remota.

Una de las características más importantes del SNM es la disminución del uso de fluoroscopia para el paciente y el operador $^{14,15}$. El catéter de ablación magnético se puede manipular de forma segura sin riesgo de causar perforaciones. Para localizar el catéter puede usarse un sistema de cartografía 3D integrado (CARTO RMT, Biosense Webster Inc.). La manipulación del catéter no necesita fluoroscopia continua y se emplea generalmente sólo para confirmar la localización de la punta antes de la aplicación. A pesar de los tiempos más largos de ablación utilizando SNM, el tiempo de fluoroscopia es considerablemente más corto en comparación con la ablación manual; se describen diferencias de hasta $29 \mathrm{~min}$ de fluoroscopia. Por otra parte, el tiempo de fluoroscopia para la ablación de fibrilación auricular guiada magnéticamente, disminuye una vez se ejecutan más procedimientos SNM ${ }^{16}$.

La ablación con catéter como tratamiento para la fibrilación auricular se asocia con riesgo de complicaciones ${ }^{17}$. Sin embargo, la seguridad de este procedimiento ha aumentado considerablemente por la introducción de SNM. Hasta la fecha se ha evaluado este problema y SNM parece mejorar la seguridad de los procedimientos de ablación de fibrilación auricular ${ }^{18}$. No obstante, debe tenerse en cuenta que estos estudios incluyeron un número limitado de pacientes y son de naturaleza retrospectiva. Con el fin de entender realmente el efecto sobre la seguridad usando SNM, se deben ejecutar más ensayos aleatorios. Los primeros informes sobre SNM en fibrilación auricular utilizando catéteres de ablación no irrigados, describieron la formación de carbón en la punta del catéter después de ablación en la aurícula izquierda; adicionalmente, algunos pacientes presentaron formación de trombos y eventos embólicos ${ }^{19}$.

La introducción de la primera generación de catéteres irrigados (SNM Navistar RMT Thermocool, Biosense Webster Inc.) mejoró el rendimiento del catéter, aunque se reportaron algunos casos de carbonización. Una vez que los catéteres irrigados magnéticos de segunda generación estuvieron disponibles, este problema se resolvió, y no hubo carbonización de la punta ni eventos embólicos relacionados ${ }^{20}$.

En informes de evaluación de SNM en la ablación de fibrilación auricular, se describe la presencia de taponamiento cardiaco como complicación del procedimiento. Sin embargo, estos traumas eran subagudos y probablemente relacionados con ablaciones prolongadas y no con la perforación aguda con la punta del catéter guiado por SNM. Recientemente, algunos datos sugieren que SNM está relacionado con cambios en la temperatura del esófago y lesiones esofágicas agudas. Todas estas lesiones esofágicas demostraron remisión completa dentro de los 14 días y fueron comparables para SNM y los enfoques convencionales de ablación ${ }^{21}$. Este mismo grupo evaluó el sistema robótico Sensei ${ }^{\circledR}$ (Hansen Medical, CA, EE.UU.) y observó una incidencia significativamente mayor de lesiones térmicas esofágicas en comparación con los procedimientos convencionales. En 
general, no se han reportado complicaciones importantes relacionadas directamente con SNM. Se ha informado hematoma en el lugar de acceso como complicación de SNM, aunque esto es comparable con procedimientos manuales.

Varios grupos que valoraron el uso de SNM en ablación de fibrilación auricular informaron capacidades ventajosas respecto al contacto del tejido, la estabilidad posicional, la maniobrabilidad y la seguridad del catéter dentro del procedimiento ${ }^{22}$. El catéter de ablación puede ser fácil de navegar dentro de la aurícula y no está restringido por cualquier curva predefinida. Debido a la porción distal flexible de la sonda que se alinea con el vector magnético, no hay restricciones para su navegación y es posible llegar a las áreas anatómicas complejas con relativa facilidad. Estas capacidades mejoradas de navegación pueden dar lugar a mejores lesiones circunferenciales de las venas pulmonares y lesiones lineales en la aurícula izquierda.

Después de la introducción de los catéteres de ablación irrigados para SNM, varios estudios evaluaron la eficacia del SNM en la fibrilación auricular paroxística. Luego de resumir los datos disponibles, los autores concluyeron que las buenas tasas de éxito agudas se lograron usando SNM, y que éstas se mantuvieron en el tiempo. Toda vez que se completaron los procedimientos, el aislamiento de venas pulmonares se alcanzó en el $96 \%$ de los pacientes. Durante un período de seguimiento medio de 11,6 meses, el $76,3 \%$ de los pacientes se mantuvieron libres de recurrencia. Como los estudios anteriores lo demostraron, estos resultados con el uso SNM, son comparables con la técnica convencional ${ }^{23}$. Los estudios previos eran no aleatorizados y por tanto se precisan ensayos clínicos aleatorizados y prospectivos para comparar la eficacia entre SNM y ablación manual de fibrilación auricular.

El SNM permite una mejor alineación perpendicular de la punta del catéter, lo que mejora la entrega de energía. Debido a un vector magnético constante, se obtiene una estabilidad óptima y la ubicación del catéter de ablación no cambia durante una aplicación. En localizaciones anatómicas complejas, tales como la válvula mitral y el miocardio trabeculado, este contacto de pared y estabilidad mejorados conducirán a un mejor suministro de energía ${ }^{24}$.

Para SNM se ha descrito una mayor duración total de la aplicación de corriente de radiofrecuencia ${ }^{25}$. Para que las lesiones de ablación sean igualmente eficaces, tiene que ser entregada más corriente de radiofrecuencia en comparación con el enfoque convencional. En estudios previos, se sugirió que el SNM no era suficientemente eficaz en la fabricación de líneas de ablación ${ }^{26}$, afirmación que se basó en el uso de un catéter de ablación SNM no irrigado de 4 u $8 \mathrm{~mm}$. Sin embargo, estos resultados durante el uso de un catéter irrigado para SNM y con tiempos de aplicación más largos de radiofrecuencia sugieren que SNM requiere un tiempo de aplicación total mayor que los procedimientos manuales y por lo tanto menos eficaces en la creación de lesiones lineales, pese a lo cual el resultado a largo plazo es equivalente.

El catéter del SNM se mantiene estable a pesar de la anatomía atrial compleja o de los movimientos cardiorrespiratorios. En contraste con los catéteres manuales, que se pueden anclar en el tejido mediante la aplicación de par de torsión al catéter, el catéter SNM tiene una fuerza de contacto inferior pero constante que se traduce en menos deformación del tejido causado por la fuerza magnética aplicada. La fuerza de contacto aplicada por el sistema de Stereotaxis SNM en la superficie endocárdica es aproximadamente de 10 a $15 \mathrm{~g}$. La estabilidad magnética del catéter proporciona un contacto de pared constante de la punta con una menor variación en las fuerzas de contacto, mientras que las técnicas convencionales muestran fuerzas de contacto intermitentes o variables. La formación de la lesión es una función de la fuerza de contacto y la energía aplicada. Sin embargo, se ha demostrado que la mayor estabilidad combinada con la fuerza de contacto inferior puede producir lesiones eficaces ${ }^{27}$. Esta disminución de la variación de la fuerza de contacto creará más trasmuralidad y lesiones de mayor volumen a fuerzas comparables. Con el fin de crear lesiones similares utilizando catéteres manuales convencionales, se requieren fuerzas mayores. Esto significa que para una fuerza dada, el SNM tiene mejor suministro de energía en comparación con los catéteres manuales. Sin embargo, a pesar de estas observaciones, la ablación de fibrilación auricular guiada por SNM está relacionada con un total de tiempos de aplicación más largos. Lo más probable es que existan regiones difíciles donde el SNM no pueda proporcionar la suficiente fuerza de contacto, y por ende haya una entrega de energía más baja. Estas regiones son exactamente las mismas que son las más difíciles de alcanzar utilizando catéteres de ablación manuales. La diferencia significativa es que hasta ahora con SNM, los autores no han tenido opciones para ajustar y mejorar la fuerza de contacto y aumentar el tamaño de la lesión. En estos casos, hay tres opciones: usar tiempos de aplicación más largos, ya que los autores saben que la lesión de radiofrecuencia no deja de crecer después de 30 segundos $^{28}$, mayor potencia de salida o cambiar a un catéter de ablación controlado manualmente.

Aunque el uso de SNM es muy intuitivo con una curva de aprendizaje rápida, está relacionado con tiempos de procedimiento más largos de ablación de fibrilación auricular. En un principio, se pensaba que esto se explicaba por la curva de aprendizaje de la nueva tecnología. Sin embargo, incluso los datos recientes sugieren que los tiempos de procedimiento son 35 - 60 minutos más largos con SNM. Además, se describe que los tiempos de ablación, definidos como el tiempo desde el primero hasta el último punto de ablación, son mayores con el SNM, hecho que podría explicarse por una velocidad de navegación más lenta en la aurícula izquierda y otras cámaras usando un SNM en comparación con navegantes manuales experimentados. Los movimientos independientes de los cambios en el vector magnético, los movimientos de los dos imanes y posteriormente el movimiento del catéter, incrementarán el tiempo dedicado a la navegación del catéter y por tanto el tiempo del procedimiento de ablación.

Al evaluar esta tecnología, los autores se han dado cuenta de algunas limitaciones relacionadas con el sistema, en comparación con la ablación con catéter convencional. En primer lugar, el uso del sistema magnético puede crear un flujo de trabajo menos eficiente. Cuando un catéter de mapeo circular se utiliza en las venas pulmonares, el operador de este sistema tiene que salir de la sala de control y entrar a la sala de procedimiento con el fin de manipular manualmente este catéter de mapeo. Dado que la manipulación del catéter se hace varias veces, podría consumir más 
tiempo que durante un procedimiento de ablación manual. Además, la manipulación del catéter de ablación magnético podría ser más lenta en comparación con los catéteres controlados manualmente, lo que conlleva tiempos de procedimiento más largos. El cambio en la alineación de la punta del catéter proporcionando el vector deseable por los imanes, lleva algún tiempo también, y los movimientos hacia adelante y atrás se hacen por separado a través de un dispositivo separado. Además, con el fin de hacer uso del SNM, se requiere hardware costoso y catéteres de ablación diseñados especialmente para los procedimientos.

\section{Desarrollos nuevos: un paso más hacia soluciones plenamente remotas}

Recientemente, se han llevado a cabo nuevos adelantos para hacer que el procedimiento de ablación sea totalmente controlado a distancia. Los resultados óptimos de la ablación de fibrilación auricular con el SNM requieren manipulaciones manuales del catéter de cartografía o mapeo circular. Aunque algunos grupos realizan la ablación de fibrilación auricular sin el uso de un catéter de mapeo circular. Hasta hace poco, no era posible navegar el catéter de cartografía circular desde la estación de trabajo remota del SNM y sólo podía hacerse manualmente.

Ahora, se ha introducido el sistema Vdrive ${ }^{\circledR}$ (Stereotaxis Inc.) para permitir la manipulación remota de catéteres de diagnóstico especializados. El operador en la estación de trabajo remota utiliza un mando a distancia para manipular los movimientos del catéter, el cualpuede hacerse avanzar, retraerse, girar y/o desviarse. El tamaño del lazo del catéter de mapeo circular puede ser modificado también. El Vdrive ${ }^{\circledR}$ puede usarse para la navegación entre las venas pulmonares, la cartografía de las cámaras y la identificación de brechas con el aislamiento segmentario.

La experiencia inicial de éste (Vdrive ${ }^{\circledR}$ ) en 94 pacientes, demostró que su uso es factible y seguro para la ablación de arritmias auriculares. En la primera serie clínica publicada para evaluar Vdrive ${ }^{\circledR}$, el $100 \%$ de los pacientes alcanzaron el punto final clínico de aislamiento pulmonar completo. En sólo el 3,2\% de los casos se requirió cambio a tecnología manual (en todos para la vena pulmonar inferior derecha). Ya que este fue el primer modelo de Vdrive ${ }^{\circledR}$, se necesitan más avances para mejorar aún más la manipulación del catéter. El Vdrive ${ }^{\circledR}$ es una herramienta muy prometedora para hacer ablación de fibrilación auricular totalmente a distancia y parece ser útil para reducir los tiempos de procedimiento. Sin embargo, se necesita más investigación para evaluar el verdadero valor de este sistema.

\section{Comentarios de expertos y visión a cinco años}

Para entender por completo los últimos desarrollos y definir el curso, los autores llevan a cabo estudios sobre nuevas mejoras y buscan entender los principios básicos de robots y robótica. La introducción de robots en la ablación con catéter tendría sentido sólo si estas máquinas abordaran los retos más importantes en la Electrofisiología cardíaca clínica. Estos desafíos incluyen: flujo de trabajo ineficiente; enormes diferencias entre operadores; problemas de seguridad y eficacia subóptima. Además, puesto que el robot se define como un agente artificial que puede hacer las tareas por su cuenta, se propone como necesario un paso más allá del nivel de los manipuladores en la introducción de robots para su verdadero uso.

Se plantea entonces una pregunta muy lógica: ¿Cuáles son los principales obstáculos para el desarrollo de verdaderos robots cardíacos? Obviamente, los retos antes mencionados no se han definido claramente desde el principio. Todo el sistema se ha utilizado a menudo más como un juguete en lugar de una herramienta real, a pesar del hecho de que el aspecto de la seguridad (en especial la dramática reducción de la exposición de fluoroscopia y el nivel de perforación cardiaca casi cero) lo hacen muy atractivo desde un principio.

Además, parece que después del entusiasmo inicial, se ha desarrollado cierto nivel de preocupación, debido, quizá, a la idea de que el proyecto de desarrollar aún más la tecnología robótica no está bien planeado. Los objetivos en la investigación van por diferentes caminos. Un ejemplo es la introducción primero de algunas funciones automáticas tales como la cartografía o el mapeo automatizados en la aurícula izquierda, mucho antes del logro de soluciones totalmente a distancia. Hay autores que piensan que el siguiente paso sin duda debería ser el desarrollo de flujo de trabajo totalmente a distancia antes de la introducción de una serie de características automatizadas, pero no hay acuerdo en las diferentes casas comerciales de desarrollo de estos productos.

En este contexto, una mejora significativa es el uso de varias herramientas (que estarán disponibles en el mercado muy pronto), entre las que cabe resaltar la utilización de un brazo robótico adicional para la manipulación remota de catéteres de diagnóstico y vainas introductoras, así como un avance significativo en la velocidad del tiempo de respuesta de los sistemas magnéticos.

Otra novedad que debe tener lugar pronto es el mejor control de suministro de energía de radiofrecuencia. En una situación ideal, en la próxima generación de catéteres magnéticos debe incluirse un sensor de fuerza de contacto, así como visualización de las lesiones de radiofrecuencia.

A pesar de todo el trabajo realizado hasta ahora, el efecto de los procedimientos de ablación SNM con catéter irrigado abierto en la reducción de las tasas de fibrilación auricular y en el logro de aislamiento de venas pulmonares, no está demostrado estadísticamente.

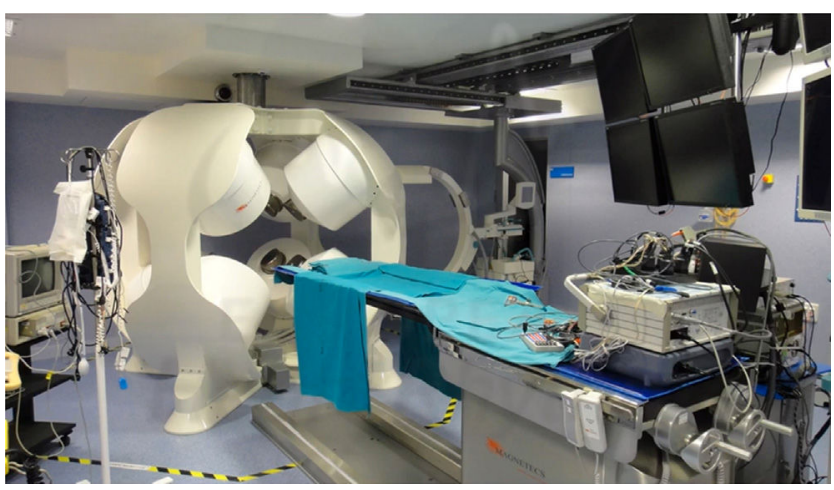

Figura 6 Sistema CGCI-Maxwell ${ }^{\circledR}$ (Catheter Guidance Control and Imaging). Cortesia Hospital Universitario La Paz. Madrid España. Dr. JL Merino. 
Tabla 1 Visión de conjunto de las características de los cuatro mayores sistemas de navegación remota

\begin{tabular}{|c|c|c|c|c|}
\hline & $\begin{array}{l}\text { Amigo }^{\circledR} \text { (Catheter } \\
\text { Robotics) }\end{array}$ & $\begin{array}{l}\text { Sensei }^{\circledR} \text { (Hansen } \\
\text { Medical) }\end{array}$ & Niobe $^{\circledR}$ (Stereotaxis) & $\begin{array}{l}\mathrm{CGCl}^{-M a x w e l l}{ }^{\circledR} \\
\text { (Magnetecs) }^{\text {Magn }}\end{array}$ \\
\hline Principio de acción & $\begin{array}{l}\text { Sistema de } \\
\text { manipulación } \\
\text { robótico de } \\
\text { catéteres }\end{array}$ & Vaina robótica & Magnetos permanentes & Electromagnetos \\
\hline $\begin{array}{l}\text { Condiciones en la } \\
\text { sala de } \\
\text { procedimientos }\end{array}$ & $\begin{array}{l}\text { Portátil } \\
\text { No requerimientos } \\
\text { especiales }\end{array}$ & $\begin{array}{l}\text { Portátil } \\
\text { No requerimientos } \\
\text { especiales }\end{array}$ & $\begin{array}{l}\text { Instalación fija } \\
\text { Refuerzo de la } \\
\text { estructura del piso } \\
\text { Blindaje magnético de la } \\
\text { sala }\end{array}$ & $\begin{array}{l}\text { Instalación fija } \\
\text { Refuerzo de la } \\
\text { estructura del piso } \\
\text { No requiere blindaje } \\
\text { magnético de la sala }\end{array}$ \\
\hline $\begin{array}{l}\text { Disponibilidad } \\
\text { actual }\end{array}$ & $\begin{array}{l}\text { No aún (solo } \\
\text { investigación) }\end{array}$ & $\mathrm{Si}$ & $\mathrm{Si}$ & $\begin{array}{l}\text { No aún (solo } \\
\text { investigación) }\end{array}$ \\
\hline Aplicaciones & $\begin{array}{l}\text { Potencialmente } \\
\text { todas las cámaras } \\
\text { cardíacas para } \\
\text { mapeo y ablación } \\
\text { Potencial para } \\
\text { mapeo y ablación } \\
\text { endocárdica y } \\
\text { epicárdica }\end{array}$ & $\begin{array}{l}\text { Taquicardia } \\
\text { supraventricular, } \\
\text { ablación de } \\
\text { fibrilación } \\
\text { auricular } \\
\text { Limitado a mapeo } \\
\text { y ablación atrial } \\
\text { Sólo endocárdico }\end{array}$ & $\begin{array}{l}\text { Estudios publicados en } \\
\text { taquicardia } \\
\text { supraventricular, } \\
\text { fibrilación auricular, } \\
\text { taquicardia ventricular } \\
\text { Todas las cámaras } \\
\text { cardíacas para mapeo } \\
\text { y ablación } \\
\text { Mapeo y ablación } \\
\text { endocárdica y epicárdica } \\
\text { Aproximación retrógrada } \\
\text { para aislamiento de } \\
\text { venas pulmonares } \\
\text { Implante de electrodos } \\
\text { de terapia de } \\
\text { resincronización } \\
\text { cardiaca en el seno } \\
\text { coronario } \\
\text { Intervenciones } \\
\text { coronarias }\end{array}$ & $\begin{array}{l}\text { Potencial para mapeo } \\
\text { y ablación de todas las } \\
\text { cámaras cardíacas } \\
\text { Potencial para mapeo y } \\
\text { ablación endocárdica } \\
\text { y epicárdica } \\
\text { Potencial para implante } \\
\text { de electrodos de terapia } \\
\text { de resincronización } \\
\text { cardiaca en el seno } \\
\text { coronario e } \\
\text { intervenciones } \\
\text { coronarias }\end{array}$ \\
\hline Ventajas & $\begin{array}{l}\text { Uso con cualquier } \\
\text { sistema de mapeo } \\
\text { tridimensional }\end{array}$ & $\begin{array}{l}\text { Estabilidad } \\
\text { del catéter } \\
\text { Uso con cualquier } \\
\text { sistema de mapeo } \\
\text { tridimensional }\end{array}$ & $\begin{array}{l}\text { Bajo riesgo } \\
\text { de perforación } \\
\text { Mapeo semiautomático } \\
\text { Numerosos estudios } \\
\text { clínicos publicados }\end{array}$ & $\begin{array}{l}\text { Verdadero sistema } \\
\text { de asa cerrada servo } \\
\text { asistido } \\
\text { Integración plena con los } \\
\text { sistemas 3D } \\
\text { Movimiento de catéteres } \\
\text { cerca al tiempo real } \\
\text { Estabilidad del catéter } \\
\text { Bajo riesgo de } \\
\text { perforación } \\
\text { Mapeo automático } \\
\text { y semiautomático }\end{array}$ \\
\hline Limitaciones & $\begin{array}{l}\text { Restringido } \\
\text { a catéteres } \\
\text { Biosense Webster } \\
\text { y Boston Scientific }\end{array}$ & $\begin{array}{l}\text { Diámetro externo } \\
\text { de la vaina y } \\
\text { longitud (no } \\
\text { acceso } \\
\text { a ventrículos } \\
\text { ni a SC) } \\
\text { No restricción de } \\
\text { catéteres }\end{array}$ & $\begin{array}{l}\text { No en pacientes con } \\
\text { dispositivos implantados } \\
\text { No movimiento de } \\
\text { catéter en tiempo real } \\
\text { Restringido a catéteres } \\
\text { magnéticos }\end{array}$ & $\begin{array}{l}\text { No en pacientes con } \\
\text { dispositivos implantados } \\
\text { Restringido a catéteres } \\
\text { magnéticos }\end{array}$ \\
\hline $\begin{array}{l}\text { Direcciones } \\
\text { futuras }\end{array}$ & $\begin{array}{l}\text { Evaluación clínica } \\
\text { Expansión hacia } \\
\text { otras marcas de } \\
\text { catéteres }\end{array}$ & $\begin{array}{l}\text { Vainas más } \\
\text { pequeñas } \\
\text { Expandir su uso a } \\
\text { todas las cámaras } \\
\text { cardíacas }\end{array}$ & $\begin{array}{l}\text { Automapeo } \\
\text { Tecnología de catéteres }\end{array}$ & $\begin{array}{l}\text { Evaluación clínica } \\
\text { Tecnología de catéteres }\end{array}$ \\
\hline
\end{tabular}


Además del sistema antes mencionado, en el mercado hay otro sistema de navegación magnética, con los mismos principios de operación, pero con una cantidad mucho mayor de imanes, lo que teóricamente se traduce en un mayor control aun del catéter dentro de la cámara cardiaca objeto de ablación ${ }^{29}$ (fig. 6).

En la tabla 1 se presenta una visión general de comparación entre los cuatro sistemas expuestos en el artículo.

\section{Conclusión}

El beneficio neto y la eficacia de la ablación guiada con sistema SNM con un catéter irrigado abierto en comparación con la navegación manual, no es fácil de predecir y no puede distinguirse claramente debido al sesgo. Los resultados están limitados por los ensayos existentes, que son pequeños y no aleatorizados, y la heterogeneidad impide conclusiones firmes. Los sistemas SNM no han mostrado ser superiores a la navegación con control manual, en el control de recurrencias de fibrilación auricular después de un período de seguimiento de 6-12 meses, pero parecen estar asociados con menos complicaciones y disminución de la exposición a la radiación ${ }^{30}$.

Sin embargo, con el fin de obtener evidencia más fuerte, es necesario diseñar un estudio aleatorizado en el que se evalúen los dos procedimientos por medio del seguimiento de un protocolo predefinido.

\section{Conflicto de intereses}

Los autores declaran no tener ningún conflicto de intereses.

\section{Bibliografía}

1. January C, Wann LS, Alpert JS, et al. 2014 AHA/ACC/HRS Guideline for the Management of Patients With Atrial Fibrillation: Executive Summary. A Report of the American College of Cardiology/American Heart Association Task Force on Practice Guidelines and the Heart Rhythm Society. J Am Coll Cardiol. 2014;64:2246-80.

2. Di Biase L, Fahmy TS, Patel D, et al. Remote magnetic navigation: human experience in pulmonary vein ablation. J Am Coll Cardiol. 2007;50:868-74.

3. Cappato R, Negroni S, Pecora D, et al. Prospective assessment of late conduction recurrence across radiofrequency lesions producing electrical disconnection at the pulmonary vein ostium in patients with atrial fibrillation. Circulation. 2003;108:1599-604.

4. Pappone C, Vicedomini G, Manguso F, et al. Robotic magnetic navigation for atrial fibrillation ablation. J Am Coll Cardiol. 2006;47:1390-400.

5. Kuck KH, Reddy VY, Schmidt B, et al. A novel radiofrequency ablation catheter using contact force sensing: Toccata study. Heart Rhythm. 2012;9:18-23.

6. Okumura $\mathrm{Y}$, Johnson SB, Bunch $\mathrm{TJ}$, et al. A systematical analysis of in vivo contact forces on virtual catheter tip/tissue surface contact during cardiac mapping and intervention. J Cardiovasc Electrophysiol. 2008;19:632-40.

7. Pappone C, Manguso F, Vicedomini G, et al. Prevention of iatrogenic atrial tachycardia after ablation of atrial fibrillation: a prospective randomized study comparing circumferential pulmonary vein ablation with a modified approach. Circulation. 2004;110:3036-42.
8. Cappato R, Calkins H, Chen SA, et al. Updated worldwide survey on the methods, efficacy, and safety of catheter ablation for human atrial fibrillation. Circ Arrhythm Electrophysiol. 2010;3:32-8.

9. Dagres N, Hindricks G, Kottkamp H, et al. Complications of atrial fibrillation ablation in a high-volume center in 1,000 procedures: still cause for concern? J Cardiovasc Electrophysiol. 2009;20:1014-9.

10. Wutzier A, Wolter T, Haverkamp W. Robotic ablation of atrial fibrillation. J Vis Exp. 2015:52560, http://dx.doi.org/10. $3791 / 52560$

11. Hlivak $P$, Mlcochova $H$, Peichl $P$, et al. Robotic navigation in catheter ablation for paroxysmal atrial fibrillation: midterm efficacy and predictors of postablation arrhythmia recurrences. J Cardiovasc Electrophysiol. 2010;22:534-40.

12. Willems S, Steve D, Servatius H, et al. Persistence of pulmonary vein isolation after robotic remote-navigated ablation for atrial fibrillation and its relation to clinical outcome. J Cardiovasc Electrophysiol. 2010;21:1-6.

13. Duncan E, Liew R, Goromonzi F, et al. A randomized controlled trial of catheter ablation of af comparing manual and robotic navigation. Heart Rhythm. 2010;7:339.

14. Bauernfeind T, Akca F, Schwagten B, et al. The magnetic navigation system allows safety and high efficacy for ablation of arrhythmias. Europace. 2011;13:1015-21.

15. Kim AM, Turakhia M, Lu J, et al. Impact of remote magnetic catheter navigation on ablation fluoroscopy and procedure time. Pacing Clin Electrophysiol. 2008;31:1399-404.

16. Lüthje L, Vollmann D, Seegers J, et al. Remote magnetic versus manual catheter navigation for circumferential pulmonary vein ablation in patients with atrial fibrillation. Clin Res Cardiol. 2011;100:1003-11.

17. Cappato R, Calkins H, Chen SA, et al. Prevalence and causes of fatal outcome in catheter ablation of atrial fibrillation. J Am Coll Cardiol. 2009;53:1798-803.

18. Choi MS, Oh YS, Jang SW, et al. Comparison of magnetic navigation system and conventional method in catheter ablation of atrial fibrillation: is magnetic navigation system is more effective and safer than conventional method? Korean Circ J. 2011;41:248-52.

19. Chun KR, Wissner E, Koektuerk B, et al. Remote-controlled magnetic pulmonary vein isolation using a new irrigated-tip catheter in patients with atrial fibrillation. Circ Arrhythm Electrophysiol. 2010;3:458-64.

20. Miyazaki S, Shah AJ, Xhaët 0 , et al. Remote magnetic navigation with irrigated tip catheter for ablation of paroxysmal atrial fibrillation. Circ Arrhythm Electrophysiol. 2010;3: 585-9.

21. Konstantinidou M, Wissner E, Chun JK, et al. Luminal esophageal temperature rise and esophageal lesion formation following remote-controlled magnetic pulmonary vein isolation. Heart Rhythm. 2011;8:1875-80.

22. Pappone C, Vicedomini G, Frigoli E, et al. Irrigated-tip magnetic catheter ablation of AF: a long-term prospective study in 130 patients. Heart Rhythm. 2011;8:8-15.

23. Sorgente A, Chierchia GB, Capulzini L, et al. Atrial fibrillation ablation: a single center comparison between remote magnetic navigation, cryoballoon and conventional manual pulmonary vein isolation. Indian Pacing Electrophysiol. J. 2010;10: 486-95.

24. Thornton AS, De Castro CA, van Deel E, et al. An in vivo comparison of radiofrequency cardiac lesions formed by standard and magnetically steered $4 \mathrm{~mm}$ tip catheters. Neth Heart J. 2010;18:66-71.

25. Solheim E, Off MK, Hoff PI, et al. Remote magnetic versus manual catheters: evaluation of ablation effect in atrial fibrillation by myocardial marker levels. J Interv Card Electrophysiol. 2011;32:37-43. 
26. Vollmann D, Lüthje L, Seegers J, et al. Remote magnetic catheter navigation for cavotricuspid isthmus ablation in patients with common-type atrial flutter. Circ Arrhythm Electrophysiol. 2009;2:603-10.

27. Burkhardt JD, Natale A. New technologies in atrial fibrillation ablation. Circulation. 2009;120:1533-41.

28. Eick OJ. Factors influencing lesion formation during radiofrequency catheter ablation. Indian Pacing Electrophysiol J. 2003;3:117-28.
29. Nguyen B, Merino JL, Gang E. Remote navigation for ablation procedures - A New step forwarding the treatment of cardiac arrhythmias. Eur Cardiol. 2010; 50-56:08-26-.

30. Proietti R, Pecoraro V, Di Biase L, et al. Remote magnetic with open-irrigated catheter vs. manual navigation for ablation of atrial fibrillation: a systematic review and meta-analysis. Europace. 2013;15:1241-8. 